\title{
Detection of Late-Time Optical Emission from SN 1941C in NGC 4136
}

\author{
Robert A. Fesen ${ }^{1}$ And Kathryn E. Weil ${ }^{1,2}$ \\ ${ }^{1} 6127$ Wilder Lab, Department of Physics and Astronomy, Dartmouth College, Hanover, NH 03755 USA \\ ${ }^{2}$ Smithsonian Astrophysical Observatory, 60 Garden Street, Cambridge, MA 02138, USA
}

\begin{abstract}
We report the detection of broad, high-velocity oxygen emission lines from the site of SN 1941C nearly eight decades after outburst, making it the oldest optically detected historical core-collapse supernova (CCSN) and one of the youngest core-collapse supernova remnants with a well determined age. In contrast to the strongly blueshifted emission line profiles observed for other late-time CCSNe thought to be due to internal dust extinction of far-side hemisphere of expanding ejecta, SN 1941C's spectrum exhibits stronger redshifted than blueshifted emissions of [O I] 6300, $6364 \AA$, [O II] 7319, $7330 \AA$, and [O III] 4959, $5007 \AA$. These oxygen emissions exhibit rest frame expansion velocities from -2200 to $+4400 \mathrm{~km} \mathrm{~s}^{-1}$. No other significant broad line emissions were detected including $\mathrm{H} \alpha$ emission. We discuss possible causes for this unusual spectrum and compare SN 1941C's optical and $\mathrm{X}$-ray luminosities to other evolved CCSNe.
\end{abstract}

Keywords: SN: individual objects: ISM: supernova remnant

\section{INTRODUCTION}

The transition of supernovae (SNe) into supernova remnants (SNRs) is an important yet poorly studied evolutionary phase (Milisavljevic \& Fesen 2017). Since SNe typically fade several magnitudes a year after outburst, detections of SNe more than one or two decades after maximum light in the optical, radio, or X-rays are rare, with nearly all late-time detections associated with core-collapse $\mathrm{SNe}(\mathrm{CCSNe})$. This has made it difficult to establish links between the properties of the few young Galactic SNRs with ages between 100 and 2000 yr, which can be studied in far greater detail than their extragalactic counterparts, with the variety of observed SN types and subclasses.

The fact that some CCSNe emitted significant emission a decade or more after outburst was only realized in the late 1980's when SN 1957D in M83 and SN 1980K in NGC 6949 were detected optically (Turatto et al. 1989; Long et al. 1989; Fesen \& Becker 1990). In those few cases where late-time SN emissions are observed, the most common interpretation is an interaction of the SN's outwardly expanding forward shock and clumpy ejecta with dense circumstellar material (CSM) (Chevalier \& Fransson 2003, 2006, 2017; Milisavljevic et al. 2012). However, other late-time energy sources such as pulsars, accretion around black holes, and magnetars have also been suggested (Chevalier \& Fransson 1994; Woosley 2010; Patnaude et al. 2011; Milisavljevic et al. 2018).

Late-time radio and X-ray emissions from shocked progenitor wind material are expected to decrease at a rate of $t^{-s}$ where $\mathrm{s}=1$ to 4 (Weiler et al. 2002; Immler,
\& Kuntz 2005; Stockdale et al. 2006), consistent with the few detections reported. In contrast, late-time optical emissions are likely generated by reverse shock-heating of dense ejecta due to the presence of a dense wind or CSM relatively close to the progenitor star (Milisavljevic et al. 2012; Black et al. 2017). Dust forming in the ejecta or in a cool dense shell of shocked SN ejecta (CDS) located between the forward CSM shock and the reverse shock front can lead to the commonly observed strongly blueshifted optical emission lines reported at late-times (Fesen \& Becker 1990; Fesen \& Matonick 1993; McCray 1993; Chevalier \& Fransson 1994; Andrews et al. 2010, 2011; Milisavljevic et al. 2012; Bevan et al. 2017) due to absorption of the redshifted, far- side of the expanding SN shell. Consequently, optical emission lines most often show strong blue/red asymmetries in lines such as $\mathrm{H} \alpha$ and [O I] 6300, $6364 \AA$ and [O III] 4959, $5007 \AA$.

Of the handful of CCSNe with reported late-time optical, X-ray, and radio emissions decades after outburst, the majority are either SN II-L or SN IIb events. These include SN 1970G in M101, SN 1979C in M100, SN 1980K in NGC 6946 and SN 1993J in M81. Such late-time emissions suggest significant CSM immediately local to the SN (Milisavljevic et al. 2012), a property not commonly present for SNe IIP, although there are exceptions (SN 2004et: Long et al. 2019). While a handful of extragalactic SNRs are have been found to be young and ejecta-dominated, such as the luminous O-rich SNR in NGC 4449 (Kirshner, \& Blair 1980; Blair et al. 1983; Long et al. 2012) and B174a in M83 (Blair et al. 2015), they are not associated with specific historical $\mathrm{SNe}$ and hence their ages and SN subtypes are not well constrained. 
At present, the oldest recorded SN exhibiting any sort of late-time emission is SN 1923A in M83. Although detected in the radio by several groups (Cowan et al. 1994; Eck et al. 1998; Stockdale et al. 2006), no identifiable X-ray or optical emission has been found (Blair \& Long 2004). Until now, the oldest SN exhibiting late-time optical and X-ray emissions is SN 1957D also in M83 (Long et al. 2012; Milisavljevic et al. 2012) but, as is common with such old historical SN, its subtype is unfortunately unknown (Long et al. 1989).

Here we present the discovery of late-time optical emissions from an even older object, namely SN 1941C. It was discovered by R.B. Jones in the southeastern outskirts of the nearly face-on $\left(i=25^{\circ}\right.$; Fridman et al. 2005) SBc spiral galaxy NGC 4136 using Harvard College photographic plates (Jones 1941). Its apparent brightness between 1941 April 16 thru 22 was between 16.8 and $17.1 \mathrm{mag}$ (Jones 1941). Although there are no published follow-up photometric or spectral observations, Adams (1941) reported that a spectrum taken by M. Humason and R. Minkowski indicated it was a Type II event.

Estimated distances to NGC 4136 vary from $8 \pm 2 \mathrm{Mpc}$ (Gusev et al. 2003), 10.5 Mpc (Bottinelli et al. 1984), and 9.7 Mpc (Tully \& Fisher 1988). Here we will adopt a distance of $9.7 \mathrm{Mpc}$. The galaxy has only a small amount of foreground Galactic extinction of $\mathrm{A}_{\mathrm{V}}=0.05$ mag (Schlafly \& Finkbeiner 2011).

Although radio searches by de Bruyn (1973) and Eck et al. (2002) for associated radio emission from historical extragalactic SNe did not survey NGC 4136, an upper limit of $4 \mathrm{mJy}$ at $2695 \mathrm{MHz}$ was reported by Brown \& Marscher (1978). However, a search of archival Chandra X-ray data covering locations of historical SN older than SN 1970G in parent galaxies closer than $15 \mathrm{Mpc}$ revealed an X-ray point source at the location of SN 1941C with a luminosity in 2002 of $\approx 5 \times 10^{37} \mathrm{erg} \mathrm{s}^{-1}$ (Soria \& Perna 2008) ${ }^{1}$.

The detection of significant X-ray flux from SN 1941C some six decades after outburst with an X-ray flux comparable to other old CCSNe (Dwarkadas \& Gruszko 2012) encouraged us to investigate possible late-time optical emission from this Type II SN. Our imaging and spectral data are described in $\S 2$, with results given in $\S 3$, with our discussion and conclusions in $\S 4$.

\section{OBSERVATIONS}

A series of $600 \mathrm{~s} \mathrm{R}$ band and $\mathrm{H} \alpha$ images of the SN 1941C site in NGC 4136 were taken on 2019 May 2 with the $2.4 \mathrm{~m}$ Hiltner telescope at MDM Observatory using the Ohio State Multi-Object Spectrograph (OSMOS, Martini et al. 2011). These were followed by a series of low-dispersion, long-slit OSMOS spectra. Using a $1.4 \operatorname{arcsec} \mathrm{N}-\mathrm{S}$ slit and a red $\mathrm{VPH} \operatorname{grism}(\mathrm{R}=$

\footnotetext{
${ }^{1}$ X-ray emission from SN 1941C can be seen in the Chandra images of NGC 4136 published by Roberts et al. 2004.
}

3000), providing a wavelength coverage $4000-8600 \AA$ and a spectral resolution of $7 \AA$, we obtained four 3000 $\mathrm{s}$ to $4000 \mathrm{~s}$ exposures which were then summed into a single 15,000 s exposure. We also obtained a $4000 \mathrm{~s}$ exposure using this same setup on the young, O-rich SNR in NGC 4449 for spectral and flux comparison purposes.

Standard pipeline data reduction of MDM images and spectra using AstroPy and $\mathrm{PYRAF}^{2}$ were performed. Spectra were reduced using the software L.A. Cosmic (van Dokkum 2001) to remove cosmic rays. Spectra were calibrated using an Ar lamp and spectroscopic standard stars (Oke 1974; Massey \& Gronwald 1990).

\section{RESULTS}

The site of SN $1941 \mathrm{C}$ in NGC 4136 is shown in Figure 1, which presents a color Sloan DSS image of the galaxy (left panel) along with MDM $\mathrm{R}$ and $\mathrm{H} \alpha$ images (center \& right panels). We found a point-like source in the $\mathrm{R}$ band image at the northern tip of a small $\left(2^{\prime \prime} \times 4^{\prime \prime}\right)$ star cluster and coincident with a faint $\mathrm{H}$ II region along the southeastern edge of the galaxy with J2000 coordinates of $\mathrm{RA}=12: 09: 21.01$, Dec $=+29: 54: 32.5$. This source is some 67 !! 1 South and 42 ". 5 East of the galaxy's nucleus, consistent with the reported offsets of $67^{\prime \prime}$ South and $44^{\prime \prime}$ East for SN $1941 \mathrm{C}$.

Figure 2 shows an optical spectrum of this point source (upper panel) taken 78 yr after SN outburst covering the wavelength region 4500 to $8000 \AA$, corrected for the $609 \mathrm{~km} \mathrm{~s}^{-1}(\mathrm{z}=0.00203)$ redshift of NGC 4136 . Broad emission lines of [O III] 4959, $5007 \AA$, [O I] 6300, $6364 \AA$, and [O II] 7319, $7330 \AA$ can be seen, along with narrow, unresolved $\mathrm{H}$ II region emission features. Measured fluxes of the broad [O I], [O II], and [O III] emissions are $1.4 \pm 0.2,1.80 \pm 0.25$, and $9.1 \pm 0.2 \times 10^{-16}$ erg s ${ }^{-1} \mathrm{~cm}^{-2}$, respectively. No broad $\mathrm{H} \alpha$ emission was detected down to a flux level of $4 \times 10^{-17} \mathrm{erg} \mathrm{s}^{-1} \mathrm{~cm}^{-2}$.

The spectrum shows redshifted [O III] emission to be brightest, extending to $5080 \AA$ or $+4400 \mathrm{~km} \mathrm{~s}^{-1}$ with a hint of possible higher velocities up to $\simeq+5000 \mathrm{~km} \mathrm{~s}^{-1}$. Blueshifted emission is also present but is much fainter and extends to much lower velocities. We observe the [O III] $4959 \AA$ line emission out only to $4922 \AA$ or -2200 $\mathrm{km} \mathrm{s}^{-1}$ (see Fig. 2, lower panel).

Figure 3 shows a comparison of emission profiles for all three forbidden oxygen emission lines in terms of expansion velocity. A dashed line marks the $+3000 \mathrm{~km}$ $\mathrm{s}^{-1}$ emission feature. Although the low $\mathrm{S} / \mathrm{N}$ of the data prevents confirmation of the full velocity range seen in the [O III] lines in the [ $\left[\begin{array}{ll}\mathrm{O} & \mathrm{I}\end{array}\right]$ and [ $\left[\begin{array}{ll}\mathrm{O} & \mathrm{II}\end{array}\right]$ lines, it is clear that the SN 1941C's redshifted, hemisphere O-rich ejecta constitutes its brightest late-time optical emission.

\footnotetext{
${ }^{2}$ PYRAF is a product of the Space Telescope Science Institute, which is operated by AURA for NASA.
} 

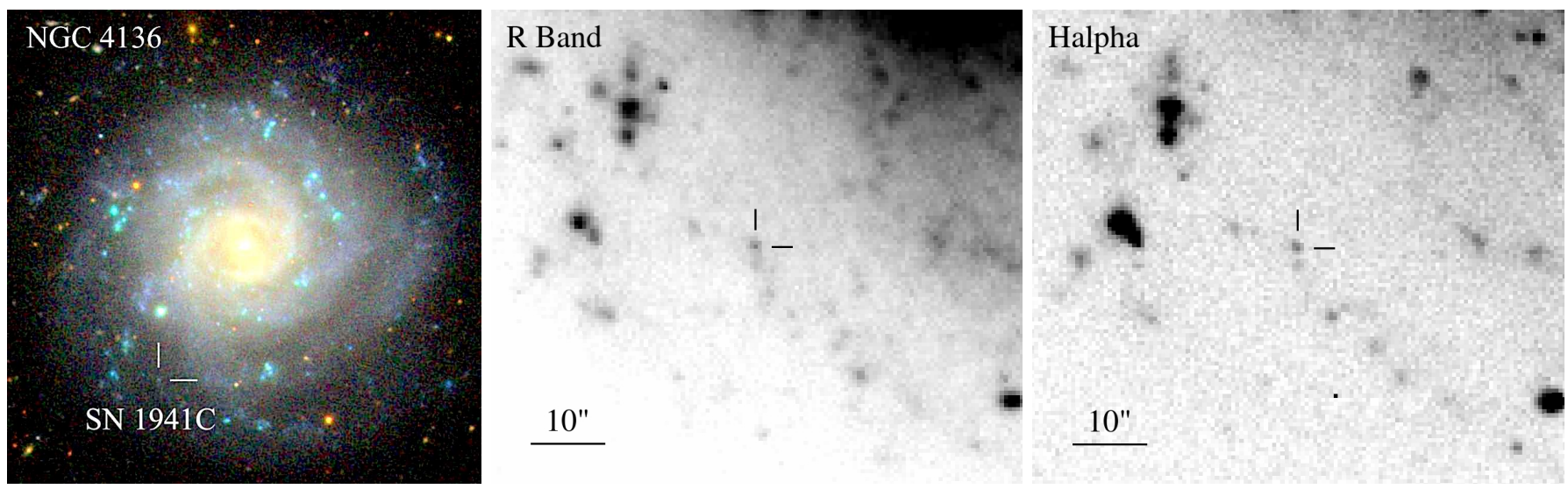

Figure 1. Left panel: Color SDSS DR14 image of NGC 4136 (credit: C. Seligman) with the location of SN $1941 \mathrm{C}$ marked. Center and right panels: MDM R band and $\mathrm{H} \alpha$ images of the SN $1941 \mathrm{C}$ site.
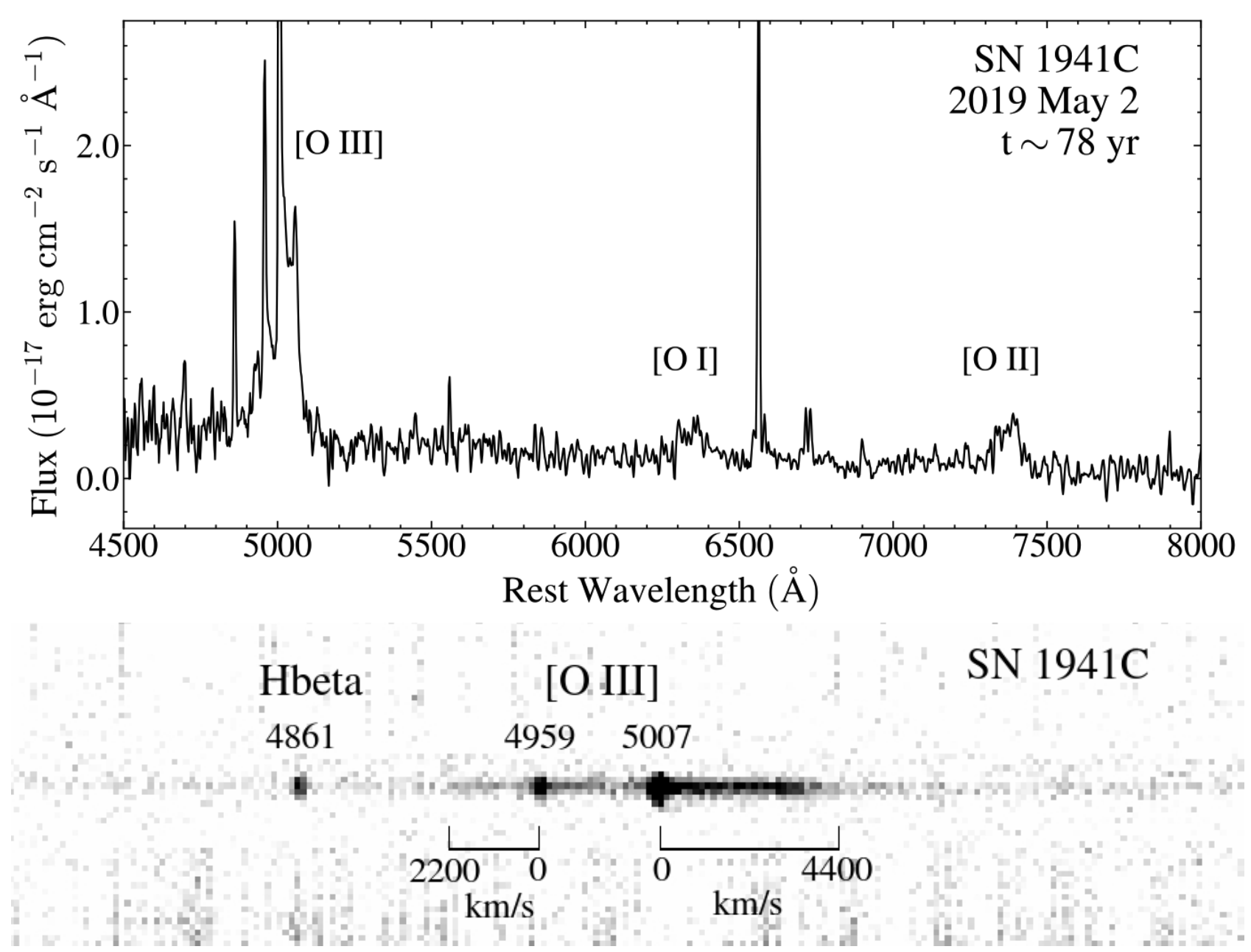

Figure 2. Upper Panel: Low dispersion spectra of SN 1941C. Lower Panel: A 2D image of the background subtracted SN $1941 \mathrm{C}$ spectrum around the [O III] 4959, $5007 \AA$ emission lines showing redshifted emission out to $4400 \mathrm{~km} \mathrm{~s}{ }^{-1}$ and fainter blueshifted emission to $-2200 \mathrm{~km} \mathrm{~s}^{-1}$. 


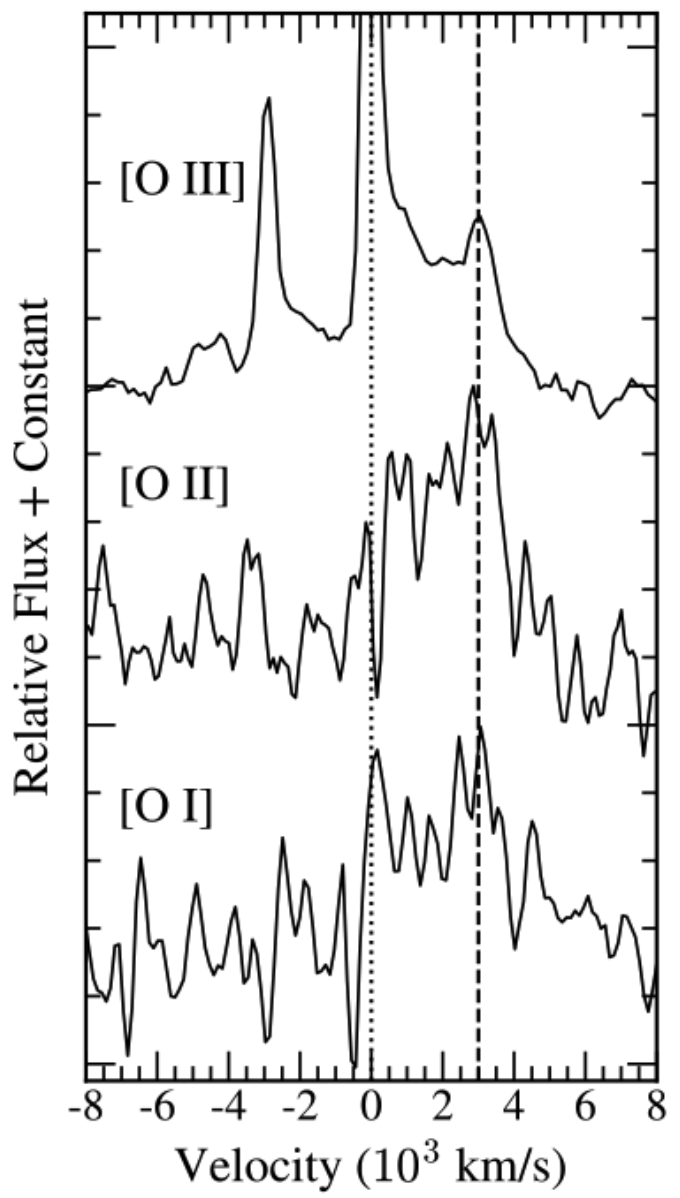

Figure 3. Plot of forbidden oxygen line emissions relative to expansion velocity. The vertical dotted line marks the location of the zero rest frame expansion velocity for [O I] $6300 \AA$, [O II] $7325 \AA$, and [O I] $5007 \AA$ line emissions. The vertical dashed line marks the emission peak seen at 3000 $\mathrm{km} \mathrm{s}^{-} 1$ in all three emission features.

Although the $3000 \mathrm{~km} \mathrm{~s}^{-1}$ emission feature is strongest in [O III] in terms of flux, the peak's relative strength appears strongest in the [O II] 7320, 7330 $\AA$ emission. This might indicate the presence of significant dust in the O-rich ejecta, like that commonly seen in late-time spectra of other CCSNe.

\section{DISCUSSION}

The site of SN 1941C is coincident with an H II region, consistent with it being a Type II SN and thus a CCSN just as Humason and Minkowski's 1941 spectrum suggested. Unfortunately, no archival Hubble Space Telescope images are available of the SN 1941C site in NGC 4136 which might inform us about the stellar neighborhood of this CCSN leading to possible estimates of its progenitor mass.

Unlike the other nearly as old historical SN, namely the $62 \mathrm{yr}$ old SN 1957D in M83 which is currently near the limit of optical detectability (Long et al. 2012; Mil- isavljevic et al. 2012), SN 1941C's late-time $78 \mathrm{yr}(\mathrm{t} \simeq$ +28 500 day) optical emission is relatively bright. While this is maybe not so surprising given its observed 2002 X-ray luminosity (Soria \& Perna 2008), SN 1941C's latetime optical spectrum is remarkable for displaying such bright redshifted emission compared to its much fainter blueshifted emission. This it is in contrast to nearly all late-time CCSNe where the SN's far-side hemisphere of ejecta emissions are strongly affected by dust which produces the observed strongly blueshifted emission profiles. Since we observed no broad $\mathrm{H} \alpha$ emission which would be the signature of dense CSM, we conclude that any dust in the SN 1941C remnant is largely confined to the ejecta, like that inferred for other CCSN exhibiting dust such as in SN 1979C (Fesen \& Matonick 1993; Milisavljevic et al. 2012).

The presence of a bright emission feature at $3000 \mathrm{~km}$ $\mathrm{s}^{-1}$ is similar to bright emission peaks off from line center seen in other late-time SN emissions, such as the SNR 4449-1 (Milisavljevic, \& Fesen 2008). Often attributed in the past to the presence of large clumps of O-rich ejecta, it is more likely due to the presence of a discrete ring(s) of ejecta such as observed in the well resolved young remnant, Cas A (DeLaney et al. 2010; Milisavljevic \& Fesen 2013). If correct, this suggests the formation of large expanding rings may be a common feature in CCSN remnants and, in turn, signal the presence of large bubble structures possibly generated by post-SN heating due to radioactive element-rich ejecta.

Table 1 shows a comparison of expansion velocities and relative optical [O I], [O III] and X-ray luminosities for several historical, extragalactic ejecta dominated SNe with known or estimated ages between 20 and 100 yr. We have also included the 350 yr old Galactic SNR, Cas A for comparison.

This table shows that, excluding the extremely bright O-rich remnant in NGC 4449, SN 1941C is quite luminous both in the optical and in X-rays for an object older than 50 years. While there is no radio detection reported for this SN, we expect it to be at least as bright as that of SN 1957D (0.6 mJy at $6 \mathrm{~cm}$ in 1998; Stockdale et al. 2006) or SNR B12-174a (0.2 mJy at $5 \mathrm{GHz}$ in 2011; Blair et al. 2015).

Table 1 also reveals again how unusual SN 1941C's blue and redshifted expansion velocities are, not in terms of absolute velocity, but in its unusual blue/red asymmetry where the far hemisphere velocity is significantly brighter than that seen for its near-side blueshifted Orich ejecta. In this respect, SN 1941C may represent a valuable transitional object where we are observing a true SNR rather than just a late evolutionary emission stage of a recent SN. Because there is no appreciable $\mathrm{H} \alpha$ emission present, the source of SN 1941C's late-time emission would not appear to be due to current or recent CSM-ejecta interaction, but more likely due to the progression of its reverse shock back into the expanding ejecta. 
Table 1. Expansion Velocities ${ }^{a}$ and Luminosities ${ }^{b}$ of Young Ejecta Dominated Core-Collapse SNRs

\begin{tabular}{|c|c|c|c|c|c|c|c|c|c|c|}
\hline \multirow[t]{2}{*}{ Object } & \multirow{2}{*}{$\begin{array}{c}\text { SN } \\
\text { Type }\end{array}$} & \multirow{2}{*}{$\begin{array}{c}\text { Host } \\
\text { Galaxy }\end{array}$} & \multirow{2}{*}{$\begin{array}{c}\text { Distance } \\
(\mathrm{Mpc})\end{array}$} & \multirow{2}{*}{$\begin{array}{l}\text { Age } \\
(\mathrm{yr})\end{array}$} & \multirow{2}{*}{$\begin{array}{c}\mathrm{V}_{\exp } \\
\left(\mathrm{km} \mathrm{s}^{-1}\right)\end{array}$} & \multicolumn{3}{|c|}{ Lum. $\left(10^{36} \mathrm{erg} \mathrm{s}^{-1}\right)$} & \multirow{2}{*}{$\begin{array}{c}\text { Obs. Epochs } \\
\text { opt:X-ray }\end{array}$} & \multirow[t]{2}{*}{ References } \\
\hline & & & & & & {$[\mathrm{O} \mathrm{I}]$} & [O III $]$ & X-ray & & \\
\hline Cassiopeia $\mathrm{A}^{c}$ & $\mathrm{IIb}$ & Milky Way & 0.0034 & $\sim 350$ & -4500 to +6500 & 0.5 & 2.3 & 27 & 1996:2012 & 1,2 \\
\hline SNR B12-174a & & M83 & 4.6 & $\leq 100$ & \pm 5200 & 6.3 & 2.5 & 7.3 & 2011:2012 & 3,4 \\
\hline SN 1941C & II & NGC 4136 & 9.7 & 78 & -2200 to +4400 & 1.7 & 11 & 50 & 2019:2002 & 5,6 \\
\hline SNR 4449-1 & .. & NGC 4449 & 3.9 & $\sim 70$ & \pm 5700 & 26 & 145 & 240 & 2019:2001 & $5,7,8,9$ \\
\hline SN 1957D & II & M83 & 4.6 & 62 & \pm 2700 & 0.3 & 1.1 & 17 & 2011:2011 & 10,11 \\
\hline SN 1970G & IIL & M101 & 7.4 & 49 & -6000 to +2200 & 3.0 & 10 & 41 & 2010:2011 & 11,12 \\
\hline SN 1979C & IIL & M100 & 15 & 40 & -6100 to +5000 & 120 & 260 & 650 & $2016: 2007$ & $11,12,13$ \\
\hline SN $1980 K$ & IIL & NGC 6946 & 5.9 & 39 & -4200 to +2100 & 2.4 & 1.6 & 30 & 2010:2004 & 11,14 \\
\hline SN 1986J & IIn & NGC 891 & 10 & 36 & -3800 to +2400 & 24 & 4.6 & 1800 & $2007: 2003$ & 11,15 \\
\hline SN 1986E & IIL & NGC 4302 & 17 & 33 & -4800 to +2600 & 47 & 10 & . & 1994: & 16 \\
\hline SN 1993J & $\mathrm{IIb}$ & M81 & 3.6 & 26 & -5800 to +7000 & 1.6 & 15 & 200 & 2009:2008 & 11,17 \\
\hline SN 1996cr & IIn & Circinus & 3.8 & 23 & \pm 4300 & 11 & 5.4 & 4000 & 2017:2007 & $11,18,19$ \\
\hline
\end{tabular}

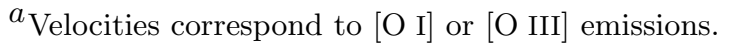

${ }^{b}$ Optical [O I] $6300+6364 \AA$, [O III] $4959+5007 \AA$, and X-ray luminosities are calculated assuming the distance listed for the host galaxy. See Dwarkadas \& Gruszko (2012) for tabulated X-ray energy range (keV) used to calculate X-ray luminosities.

${ }^{c}$ Values for Cas A are taken from Winkler et al. (2017) which include extinction corrections assuming $\mathrm{A}_{\mathrm{V}}=6.2$.

References: 1: Winkler et al. (2017); 2: Milisavljevic \& Fesen (2013); 3: Long et al. (2014); 4: Blair et al. (2015); 5: this paper; 6: Soria \& Perna (2008); 7: Milisavljevic, \& Fesen (2008); 8: Patnaude \& Fesen (2003); 9: Summers et al. (2003); 10: Long et al. (2012); 11: Milisavljevic et al. (2012); 12: Dittmann et al. (2014); 13: Patnaude et al. (2011); 14: unpublished 2016 MMT spectrum; 14: Fridriksson et al. (2008); 15: Houck (2005); 16: Cappellaro et al. (1995); 17: Chandra et al. (2009); 18: Patnaude et al. in prep; 19: Bauer et al. (2008)

In this respect, the remnant of SN $1941 \mathrm{C}$ is like that of Cas A where its total global emission is dominated by emissions from ejecta with only very faint $\mathrm{H} \alpha$ and [N II] emissions (Milisavljevic, \& Fesen 2008). Moreover, Cas A's optical emission has radially changed in the roughly 70 year observing period (1950 to present) where in the early 1950 's only a few relatively faint optical filaments along its northern limb were visible, whereas currently a nearly complete and bright ring of ejecta emissions is seen (Patnaude \& Fesen 2014). Cas A appears to also shared SN 1941C's asymmetry in its blue and red expansion velocities (see Table 1 ).

It is possible that SN 1941C's O-rich ejecta are in a similar early stage of being reverse shockheated where relatively limited portions of the object's expanding ejecta shell are visible leading to the blueshifted/redshifted asymmetry observed. Consequently, it may prove useful to monitor SN 1941C's latetime optical and X-ray emissions to observe changes in both luminosity and velocity.

The presence of an unknown amount of internal ejecta extinction, in both the facing but especially the remnant's far-side redshifted hemisphere, makes mass esti- mates based on observed emission fluxes quite uncertain. Nonetheless, a comparison of the SN 1941C remnant's [O I] and [O III] luminosities to the similar age O-rich SNe/young SNR seen in NGC 4449 (see Table 1) which has an estimated oxygen mass $\sim 7.5 \times 10^{-2} \mathrm{M}_{\odot}$ (Kirshner, \& Blair 1980) suggests an oxygen mass $\sim 10^{-2}$ $\mathrm{M}_{\odot}$. Because larger expansion velocities hinted by our low $\mathrm{S} / \mathrm{N}$ spectra may indicate a more extensive O-rich expanding shell, this value could be viewed as a lower limit.

Due to the relatively low quality and quantity of early 20th century photographic spectra of SNe and the fact that the spectral derived classes of Type I and Type II SNe was only established in 1941 (Minkowski 1941; Filippenko 1997), only about a dozen of the 40 historical extragalactic SNe with ages greater than SN 1941C have been classified or proposed as either possible or likely Type II events (Barbon et al. 2010). However, some of these assigned SN types are questionable.

Prior to the discovery of SN 1941C, Zwicky (1964) listed just five SNe (1936A, 1937A, 1940A, 1940B, and 1941A) as definitely or likely Type II SNe. Subsequent SN lists by Kowal, \& Sargent (1971), Sargent et al. 
(1974), Maza, \& van den Bergh (1976), and Flin et al. (1979) increased this number by adding four more (1919A, 1926A, 1937F, and 1939C). More recent listings such as the Asiago Supernova Catalogue (Barbon et al. 1989, 1999, 2010) added several more (1909A, 1917A, 1921A, 1923A, and 1940C) along with II-L and II-P light curve sub-classifications for some.

However, poor quality and/or the absence of spectral data have led to differences in assigned SN types in various lists for some SNe prior to $1941 \mathrm{C}$, suggesting the fidelity of some SN assigned types might not be high. An example is SN 1909A where its photographic light curve does not seem to fit either that of a Type I or II (Sandage, \& Tammann 1974; Patat et al. 1993), resulting in a variety of its assigned SN type in various catalogs: peculiar, IV, peculiar, II-P (Sargent et al. 1974; Flin et al. 1979; Barbon et al. 1989, 1999).

Another example is that of SN 1917A in NGC 6946. A $6 \mathrm{hr}$ long slitless spectrum obtained by Pease and Ritchey at least a month or so after maximum light (Ritchey 1917; Shapley 1917) was described as "a strong continuous spectrum, crossed by what appears to be a series of bright bands". A later examination of this spectrum by Baade and Humason (Baade 1938) showed "a typical supernova spectrum with the wide $\lambda 4600$ band as the dominant feature", a property common in postmax Type Ia spectra (Black et al. 2016). Although this assessment was subsequently echoed by Mayall (1948) in his report on the spectrum of the Type II SN 1948B in the same galaxy, SN 1917A is listed as a Type II in most recent SN catalogs (Barbon et al. 1999, 2010; Lennarz et al. 2012; Guillochon et al. 2017).

Consequently, until emission from more historical CCSNe older than SN 1941C are detected and studied across a range of wavelengths, our understanding of the transition of CCSNe into core-collapse SNRs is limited. The very small number of detected young extragalactic CCSNe, unfortunately, does not offer much help lluminating this evolutionary phase. Only two Magellanic Clouds core-collapse SNRs have estimated ages less than 2000 yr (namely, 1E0202-7219 and 0540-69.3) and despite extensive SNR surveys in nearby galaxies including M31, M33, M83, and NGC 6946 (Long 2017; Long et al. 2019) only two extragalactic CCSN remnants outside of the Magellanic Clouds not associated with historical SNe with estimated ages of a few hundred years or less have been found, namely, SNR 4449-1 in NGC 4449 and SNR B12-174a in M83 (see Table 1).

This situation, together with the small sample size of young, $\leq 1000$ yr old Galactic CCSN remnants (i.e., the Crab, 3C58, and Cas A), makes further study of the 78 yr old SN 1941C across multi-wavelengths especially valuable for investigating the SN-SNR connection. This could include HST multiband imaging of the explosion site and stellar association, deep Chandra imaging for X-ray spectral analysis, an investigation of its likely late-time radio emission, as well as higher $\mathrm{S} / \mathrm{N}$ optical spectra.

\section{ACKNOWLEDGMENTS}

We thank Bill Blair, Frank Winkler, and Dan Milisavljevic for sharing late-time spectral data with us, Dan Patnaude for help with the X-ray analysis of SN 1986E, David H. Roberts for his helpful discussions of VLA observations of NGC 4136, an anonymous referee for several helpful comments and corrections, and Eric Galayda and the MDM staff for assistance with our observations. R.A.F. and K.E.W acknowledge support from STScI Guest Observer Programs 15337 and 15515. K.E.W. also acknowledges support from Dartmouth's Guarini School of Graduate and Advanced Studies, and the Chandra X-ray Center under CXC grant GO7-18050X. This work is part of R.A.F's Archangel III Research Program. Finally, we would like to acknowledge the extensive research on SNe done by Milton Humason and Rudolph Minkowski which included the spectroscopic classification of SN 1941C, the subject of this paper.

Facilities: Hiltner (OSMOS), Sloan

Software: PYRAF (Green 2012), AstroPy (Astropy Collaboration et al. 2013, 2018), ds9 (Smithsonian Astrophysical Observatory 2000), L.A. Cosmic (van Dokkum 2001) 


\section{REFERENCES}

Adams, W. S. 1941, Mount Wilson Observatory Annual Report, 1940-1941

Andrews, J. E., Gallagher, J. S., Clayton, G. C., et al. 2010, ApJ, 715, 541

Andrews, J. E., Sugerman, B. E. K., Clayton, G. C., et al. 2011, ApJ, 731, 47

Astropy Collaboration, Robitaille, T. P., Tollerud, E. J., et al. 2013, A\&A, 558, A33

Astropy Collaboration, Price-Whelan, A. M., Sipőcz, B. M., et al. 2018, AJ, 156, 123

Baade, W. 1938, ApJ, 88, 285

Bauer, F. E., Dwarkadas, V. V., Brandt, W. N., et al. 2008, ApJ, 688, 1210

Bevan, A., Barlow, M. J., \& Milisavljevic, D. 2017, MNRAS, 465, 4044

Black, C. S., Milisavljevic, D., Margutti, R., et al. 2017, ApJ, 848, 5

Black, C. S., Fesen, R. A., \& Parrent, J. T. 2016, MNRAS, 462,649

Blair, W. P., Kirshner, R. P., \& Winkler, P. F. 1983, ApJ, 272,84

Blair, W. P., \& Long, K. S. 2004, ApJS, 155, 101

Blair, W. P., Winkler, P. F., Long, K. S., et al. 2015, ApJ, 800, 118

Barbon, R., Cappellaro, E., \& Turatto, M. 1989, A\&AS, 81, 421

Barbon, R., Buondí, V., Cappellaro, E., et al. 1999, A\&AS, 139, 531

Barbon, R., Buondi, V., Cappellaro, E., et al. 2010, VizieR Online Data Catalog, B/sn

Bottinelli, L., Gouguenheim, L., Paturel, G., et al. 1984, A\&AS, 56, 381

Brown, R. L., \& Marscher, A. P. 1978, ApJ, 220, 467

Cappellaro, E., Danziger, I. J., \& Turatto, M. 1995, MNRAS, 277, 106

Chandra, P., Dwarkadas, V. V., Ray, A., et al. 2009, ApJ, 699,388

Chevalier, R. A., \& Fransson, C. 1994, ApJ, 420, 268

Chevalier, R. A., \& Fransson, C. 2003, Supernovae and Gamma-ray Bursters, 171

Chevalier, R. A., \& Fransson, C. 2006, ApJ, 651, 381

Chevalier, R. A., \& Fransson, C. 2017, Handbook of Supernovae, 875

Cowan, J. J., Roberts, D. A., \& Branch, D. 1994, ApJ, 434, 128

de Bruyn, A. G. 1973, A\&A, 26, 105

DeLaney, T., Rudnick, L., Stage, M. D., et al. 2010, ApJ, 725,2038
Dittmann, J. A., Soderberg, A. M., Chomiuk, L., et al. 2014, ApJ, 788, 38

Dwarkadas, V. V., \& Gruszko, J. 2012, MNRAS, 419, 1515

Eck, C. R., Roberts, D. A., Cowan, J. J., et al. 1998, ApJ, 508,664

Eck, C. R., Cowan, J. J., \& Branch, D. 2002, ApJ, 573, 306

Fesen, R. A., \& Becker, R. H. 1990, ApJ, 351, 437

Fesen, R. A., \& Matonick, D. M. 1993, ApJ, 407, 110

Filippenko, A. V. 1997, ARA\&A, 35, 309

Flin, P., Karpowicz, M., Murawski, W., et al. 1979, Acta Cosmologica, 8, 5

Fridman, A. M., Afanasiev, V. L., Dodonov, S. N., et al. 2005, A\&A, 430, 67

Fridriksson, J. K., Homan, J., Lewin, W. H. G., et al. 2008, ApJS, 177, 465

Green, W. 2012, Society for Astronomical Sciences Annual Symposium, 31, 159

Guillochon, J., Parrent, J., Kelley, L. Z., et al. 2017, ApJ, 835,64

Gusev, A. S., Zasov, A. V., \& Kaisin, S. S. 2003, Astronomy Letters, 29, 363

Houck, J. C. 2005, X-Ray and Radio Connections, eds. L.O. Sjouwerman and K.K Dyer) Published electronically by NRAO, p 4.06

Immler, S., \& Kuntz, K. D. 2005, ApJL, 632, L99

Jones, R. B., IAU Cir. 866, 2

Kirshner, R. P., \& Blair, W. P. 1980, ApJ, 236, 135

Kowal, C. T., \& Sargent, W. L. W. 1971, AJ, 76, 756

Lennarz, D., Altmann, D., \& Wiebusch, C. 2012, A\&A, 538, A 120

Long, K. S., Blair, W. P., \& Krzeminski, W. 1989, ApJL, 340, L25

Long, K. S., Blair, W. P., Godfrey, L. E. H., et al. 2012, ApJ, 756, 18

Long, K. S., Kuntz, K. D., Blair, W. P., et al. 2014, ApJS, 212,21

Long, K. S. 2017, Handbook of Supernovae, Springer International Publishing AG, p2005

Long, K. S., Winkler, P. F., \& Blair, W. P. 2019, ApJ, 875, 85

Martini, P., Stoll, R., Derwent, M. A., et al. 2011, PASP, 123,187

Massey, P., \& Gronwald, C. 1990, ApJ, 358, 344

Mayall, N. U. 1948, PASP, 60, 266

Maza, J., \& van den Bergh, S. 1976, ApJ, 204, 519

McCray, R. 1993, ARA\&A, 31, 175

Milisavljevic, D., \& Fesen, R. A. 2008, ApJ, 677, 3061

Milisavljevic, D., Fesen, R. A., Chevalier, R. A., et al. 2012, ApJ, 751, 25 
Milisavljevic, D., \& Fesen, R. A. 2013, ApJ, 772, 134

Milisavljevic, D., \& Fesen, R. A. 2017, Handbook of

Supernovae, 2211

Milisavljevic, D., Patnaude, D. J., Chevalier, R. A., et al. 2018, ApJL, 864, L36

Minkowski, R. 1941, PASP, 53, 224

Oke, J. B. 1974, ApJS, 27, 21

Oke, J. B., \& Searle, L. 1974, ARA\&A, 12, 315

Patat, F., Barbon, R., Cappellaro, E., et al. 1993, A\&AS, 98,443

Patnaude, D. J., \& Fesen, R. A. 2003, ApJ, 587, 221

Patnaude, D. J., Loeb, A., \& Jones, C. 2011, NewA, 16, 187

Patnaude, D. J., \& Fesen, R. A. 2014, ApJ, 789, 138

Ritchey, G. W. 1917, PASP, 29, 211

Roberts, T. P., Warwick, R. S., Ward, M. J., et al. 2004, MNRAS, 349, 1193

Sandage, A., \& Tammann, G. A. 1974, ApJ, 194, 223

Sargent, W. L. W., Searle, L., \& Kowal, C. T. 1974,

Supernovae and Supernova Remnants, 33

Schlafly, E. F., \& Finkbeiner, D. P. 2011, ApJ, 737, 103

Shapley, H. 1917, PASP, 29, 213

Smithsonian Astrophysical Observatory 2000, SAOImage DS9: A utility for displaying astronomical images in the X11 window environment, ascl:0003.002
Soria, R., \& Perna, R. 2008, ApJ, 683, 767

Stockdale, C. J., Maddox, L. A., Cowan, J. J., et al. 2006, AJ, 131, 889

Summers, L. K., Stevens, I. R., Strickland, D. K., \& Heckman, T. M. 2003, MNRAS, 342, 690

Turatto, M., Cappellaro, E., \& Danziger, I. J. 1989, The Messenger, 56, 36

Tully, R. B., \& Fisher, J. R. 1988, Catalog of Nearby Galaxies

van Dokkum, P. G. 2001, PASP, 113, 1420

Weiler, K. W., Panagia, N., Montes, M. J., et al. 2002, ARA\&A, 40, 387

Winkler, P. F., Blair, W. P., \& Long, K. S. 2017, ApJ, 839, 83

Woosley, S. E. 2010, ApJL, 719, L204

Zwicky, F. 1964, Interim Report to the Committee for Research on Supernovae Within Commission 28 of the International Astronomical Union (IAU) and to the National Science Foundation 